Review

\title{
Interplay between iron homeostasis and virulence: Fur and RyhB as major regulators of bacterial pathogenicity
}

\author{
Gaëlle Porcheron ${ }^{\mathrm{a}}$, Charles M. Dozois ${ }^{\mathrm{a}, \mathrm{b}, \mathrm{c}, *}$ \\ a INRS-Institut Armand Frappier, Laval, Québec, Canada \\ ${ }^{\mathrm{b}}$ Centre de Recherche en Infectiologie Porcine et Aviaire (CRIPA), Université de Montréal, Faculté de Médecine Vétérinaire, Saint-Hyacinthe, \\ Québec, Canada \\ ${ }^{\mathrm{C}}$ Groupe de Recherche sur les Maladies Infectieuses du Porc (GREMIP), Université de Montréal, Faculté de Médecine Vétérinaire, \\ Saint-Hyacinthe, Québec, Canada
}

\section{A R T I C L E I N F O}

\section{Article history:}

Received 17 December 2014

Received in revised form 23 March 2015

Accepted 25 March 2015

\section{Keywords:}

Iron

Virulence

Fur

RyhB

Regulation

\begin{abstract}
A B S T R A C T
In bacteria-host interactions, competition for iron is critical for the outcome of the infection. As a result of its redox properties, this metal is essential for the growth and proliferation of most living organisms, including pathogenic bacteria. This metal is also potentially toxic, making the precise maintenance of iron homeostasis necessary for survival. Iron acquisition and storage control is mediated in most bacteria by the global ferric uptake regulator (Fur) and iron-responsive small regulatory non-coding RNAs (RyhB in the model organism Escherichia coli). While the role of these regulators in iron homeostasis is well documented in both pathogenic and non-pathogenic bacteria, many recent studies also demonstrate that these regulators are involved in the virulence of pathogenic bacteria. By sensing iron availability in the environment, Fur and RyhB are able to regulate, either directly or indirectly via other transcriptional regulators or modulation of intracellular iron concentration, many virulence determinants of pathogenic bacteria. Iron is thus both a nutritional and regulatory element, allowing bacteria to adapt to various host environments by adjusting expression of virulence factors. In this review, we present evidences that Fur and RyhB are the major regulators of this adaptation, as they are involved in diverse functions ranging from iron homeostasis to regulation of virulence by mediating key pathogen responses such as invasion of eukaryotic cells, toxin production, motility, quorum sensing, stress resistance or biofilm formation. Therefore, Fur and RyhB play a major role in regulating an adaptative response during bacterial infections, making them important targets in the fight against pathogenic bacteria.
\end{abstract}

(C) 2015 Elsevier B.V. All rights reserved.

\section{Contents}

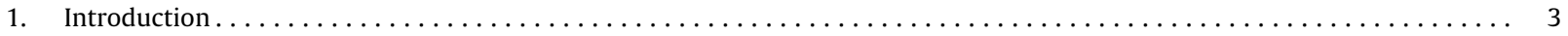

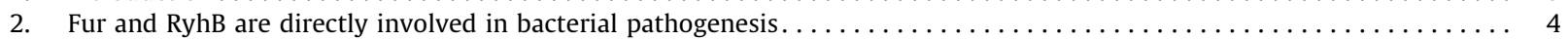

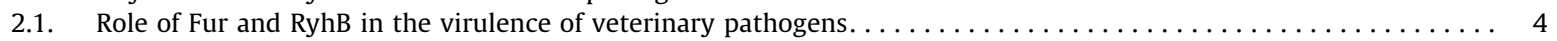

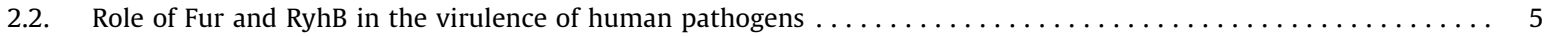

\footnotetext{
* Corresponding author at: INRS-Institut Armand Frappier, 531, Boul. des Prairies, Laval, Québec H7V 1B7, Canada. Tel.: +1 4506865515 ; fax: +1 4506865501

E-mail address: charles.dozois@iaf.inrs.ca (C.M. Dozois).
} 


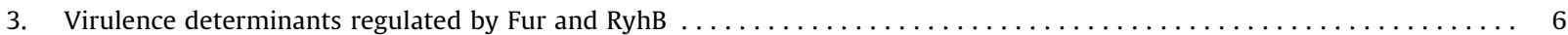

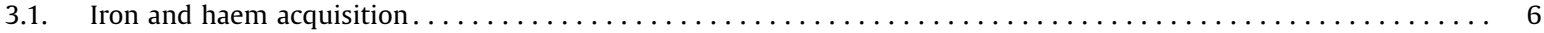

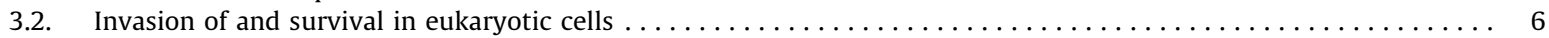

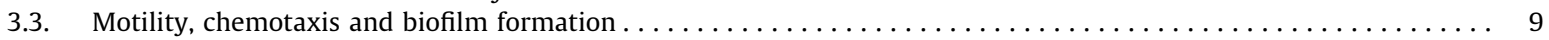

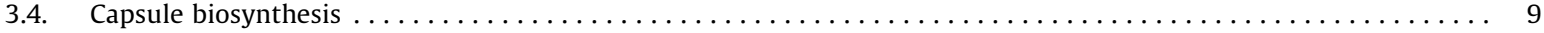

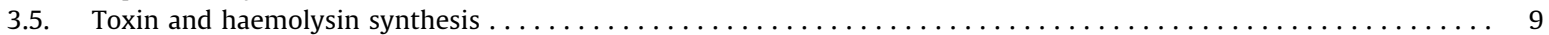

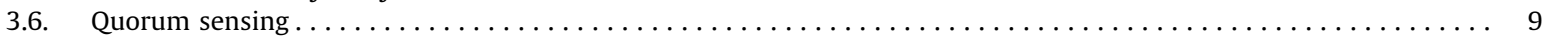

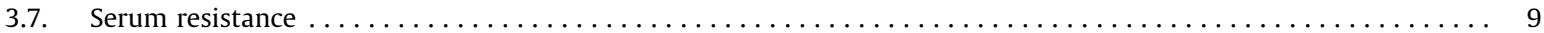

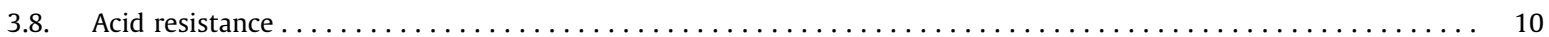

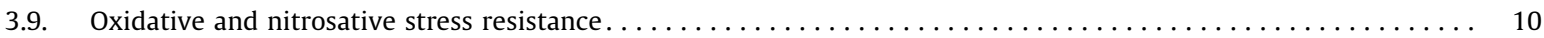

4. Fur and RyhB modes of action: from direct to indirect regulation of virulence determinants $\ldots \ldots \ldots \ldots \ldots \ldots \ldots \ldots \ldots$

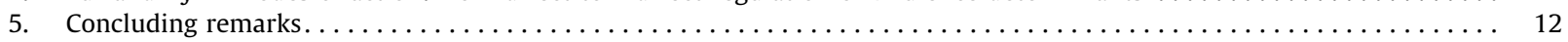

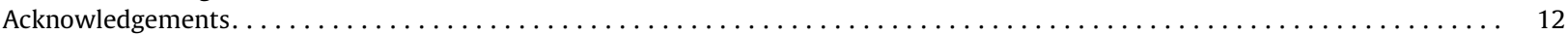

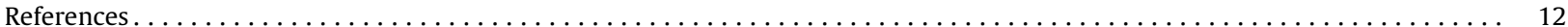

\section{Introduction}

Iron is the most abundant transition metal in the host, and this metal is an essential co-factor for many metabolic enzymes involved in biological reactions such as respiration, DNA biosynthesis, gene regulation and for tricarboxylic acid (TCA) cycle. Due to its poor solubility under aerobic conditions at neutral $\mathrm{pH}$, iron is nearly inaccessible within animal hosts. Proteins such as ferritin, haemoglobin or transferrin chelate iron and thus can restrict the availability of this essential metal from invading pathogens. In addition to extracellular metal restriction mechanisms, host cells can also deplete metals from inside phagosomes. In order to acquire iron in iron-limiting environments, bacteria synthesize and secrete highaffinity iron chelating molecules, siderophores, which contribute to bacterial survival by sequestering iron from the host (Hood and Skaar, 2012; Porcheron et al., 2013). Although iron is detrimental for survival, it is also toxic under oxygen-rich conditions. Indeed, it is involved, through the Fenton reaction, in the production of highly reactive oxygen species (ROS) that damage nucleic acids, proteins and cellular membranes (Andrews et al., 2003). Iron is thus both essential and potentially toxic for most living organisms, making the precise maintenance of iron homeostasis necessary for survival. Many organisms have thus developed strong homeostatic systems that maintain intracellular iron concentration within a range that is not detrimental for the cell.

To respond to their environment, especially iron availability, response regulators alter expression of genes that promote bacterial survival. In the model organism Escherichia coli, iron acquisition and storage are controlled by the global ferric uptake regulator (Fur) protein and the small regulatory non-coding RNA (sRNA) RyhB. Under iron-rich conditions, $\mathrm{Fe}^{2+}$-Fur acts as a negative regulator of $r y h B$ and iron uptake genes by binding in a sequencespecific manner within the promoter region of target genes, efficiently preventing their expression (Fig. 1). The Fur-binding site of regulated promoters contains a consensus of three or four imperfect adjacent hexamers 5'-GATAAT-3', although an alternative consensus sequence 5'-TGATAATNATTATCA-3' has been proposed (Baichoo and Helmann, 2002). Fur-mediated sensing of iron availability is conserved across Gram-positive and Gram-negative bacteria (Andrews et al., 2003). When iron availability is limited [below 5-10 $\mu \mathrm{M}$ external iron concentration (Andrews et al., 2003)], Fur becomes inactive and subsequently the production of RyhB and iron acquisition systems is initiated in order to restore iron homeostasis (Fig. 1) (Massé and Gottesman, 2002). The RyhB-mediated regulation is initiated by the antisense pairing of the sRNA

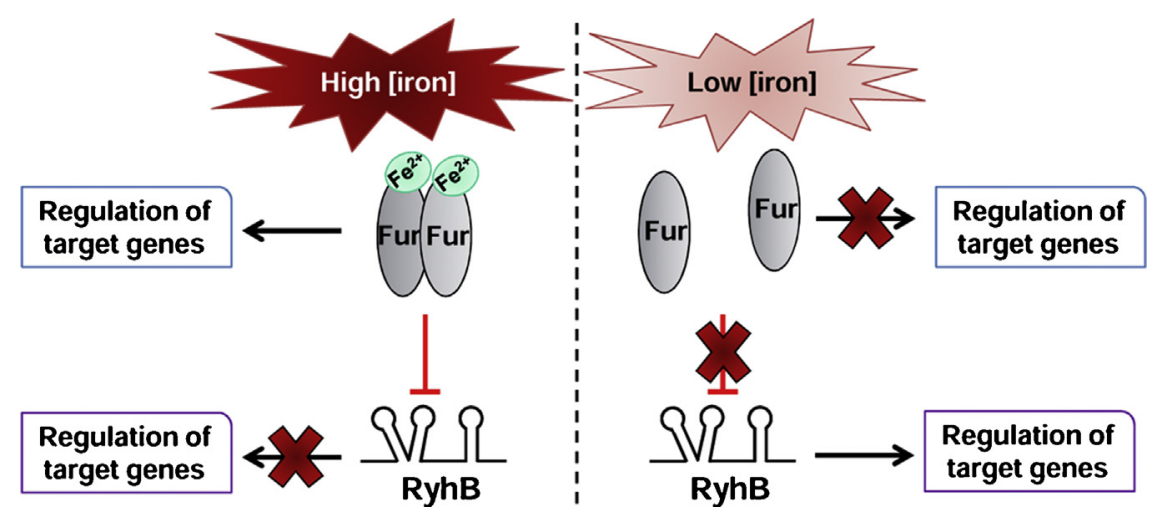

Fig. 1. Schematic representation of Fur and RyhB regulation. In iron-rich environments, the active Fur protein represses many genes as well as ryhB, resulting in deregulation of RyhB-specific target genes. In iron-poor conditions, Fur repression is relieved and ryhB is expressed, leading to regulation (activation or repression) of RyhB-specific target genes. 
with its mRNA targets to positively or negatively affect their translation and stability. Many RyhB targets are nonessential iron-using proteins such as succinate dehydrogenase, superoxide dismutase, iron-storage proteins (ferritins) and enzymes of the TCA cycle. By binding to those mRNAs, RyhB shuts down translation and stimulates their rapid degradation through the action of RNase E. RyhB thus promotes availability of iron for essential iron-using proteins required for many biological processes under iron-limited conditions (respiration, oxygen transport, DNA biosynthesis) (Massé et al., 2005). In addition to posttranscriptional gene repression, RyhB is able to promote the expression of a number of genes involved in iron homeostasis. Indeed, RyhB activates translation of shiA and cirA mRNAs by disrupting inhibitory structure that sequesters the translation initiation region, thereby favouring translation and transcript stability (Prevost et al., 2007; Salvail et al., 2013).

The first publication characterizing E. coli RyhB noted that the gene was conserved in several bacterial genera, including Salmonella, Klebsiella, Yersinia and Vibrio (Massé and Gottesman, 2002). Subsequent studies have identified RyhB in these and other species of bacteria. In Shigella flexneri and Shigella dysenteriae, the nucleic acid sequence of ryhB is identical to those in E. coli (Murphy and Payne, 2007; Oglesby et al., 2005). RyhB is less well conserved between E. coli and Vibrio cholerae, in which this sRNA is longer ( $>200$ nucleotides vs. 90 in E. coli). Homologs of this longer RyhB have also been identified in other Vibrio species such as Vibrio parahaemolyticus and Vibrio vulnificus (Mey et al., 2005a). Many other pathogens possess two RyhB homologs. Salmonella enterica possesses two sRNAs, RyhB-1 (or RfrA) and RyhB-2 (or RfrB), that are the orthologue and paralogue of RyhB, respectively. While the production of both sRNAs is influenced in response to ironavailability by the activity of Fur, production of RyhB-2 is primarily regulated by the activity of the stationary phase sigma factor RpoS (Kim and Kwon, 2013a). Yersinia pestis also encodes two RyhB homologues, RyhB-1 and RyhB-2, both regulated by Fur. It is also suggested that Klebsiella pneumoniae encodes two RyhB homologs (Oglesby-Sherrouse and Murphy, 2013). In Pseudomonas aeruginosa, the Fur-regulated PrrF sRNAs, PrrF-1 and PrrF-2, share no significant sequence homology with RyhB, but they mediate an analogous iron-sparing response. As with RyhB in E. coli, these iron-responsive sRNAs repress the expression of genes involved in iron storage. Moreover, other Pseudomonads such as Pseudomonas putida, Pseudomonas fluorescens and Pseudomonas syringae also have PrrF sRNAs (Wilderman et al., 2004). Although mainly described in Gram-negative bacteria, similar iron-responsive sRNA has also been described in Bacillus subtilis. Similar to RyhB, the sRNA FsrA is regulated by Fur and affects the expression of iron-using proteins (Helmann, 2014). In addition to regulated iron acquisition and storage, more and more studies describe the role of Fur and RyhB in regulating virulence properties of pathogenic bacteria.

Excellent reviews have recently described the regulation mechanisms of Fur and iron-responsive sRNAs (Oglesby-Sherrouse and Murphy, 2013; Salvail and Massé, 2012; Troxell and Hassan, 2013). In this review, we highlight how these iron homeostasis regulators, Fur and RyhB, are also involved in virulence of pathogenic bacteria, especially by regulating many virulence determinants. To do so, they can act either as direct or indirect regulators, by mediating targeted or broader responses. Fur and RyhB impact a broad range of virulence processes in response to iron availability in the environment, making iron a key environmental factor contributing to bacterial virulence.

\section{Fur and RyhB are directly involved in bacterial pathogenesis}

Given the essential role of iron availability for a successful infection, it is not surprising that the role of the iron homeostasis regulators Fur and RyhB in virulence have been investigated in many pathogens, whether they are involved in human or animal diseases.

\subsection{Role of Fur and RyhB in the virulence of veterinary pathogens}

Many reports highlight the importance of Fur in pathogenicity of bacteria of veterinary importance, including bacterial pathogens of fish, swine or poultry (Table 1). In Aeromonas salmonicida subsp. salmonicida, the agent of furunculosis, a systemic disease of salmonids, a fur mutant shows significantly reduced pathogenicity compared to the wild-type strain in salmon infected using an immersion technique (Ebanks et al., 2013). In a pathogenic $P$. fluorescens strain, a fur mutant exhibits impaired ability to disseminate in host blood and tissues, and drastic attenuation in overall bacterial virulence in a Japanese flounder infection model following intraperitoneal injection. Indeed, the $\mathrm{LD}_{50}$ of the fur mutant is 1000 -fold higher than that of the wild-type strain (Wang et al., 2009). In Edwardsiella ictaluri, one of the most important pathogens of channel catfish, a fur mutant is attenuated in both the zebrafish model and its natural host catfish, with a 10 -fold increase and a 1000 -fold increase of $\mathrm{LD}_{50}$ compared to the wild-type strain, respectively (Santander et al., 2012). In Edwardsiella tarda, the etiological agent of edwardsiellosis, a systemic disease of cultured fish, overexpression of fur leads to attenuation of bacterial virulence in Japanese flounder (Wang et al., 2008). In an iron-overloaded mouse model, competitive experiments with the wild-type strain have demonstrated that the ryhB mutant of $V$. vulnificus is decreased in virulence. Moreover, whereas $\mathrm{LD}_{50}$ of the fur mutant is the same as that of the wild-type strain, the $\mathrm{LD}_{50}$ of the ryhB mutant is more than 30 -fold higher than that of the wild-type strain (Alice et al., 2008). In Actinobacillus pleuropneumoniae, the causative agent of porcine pleuropneumonia, a fur mutant shows reduced virulence in an aerosol infection model of pigs (Jacobsen et al., 2005). Fur is also required for systemic infection of mice by $S$. enterica serovar Typhimurium (Troxell et al., 2011). Moreover, nonproliferating intracellular $S$. Typhimurium upregulate RyhB-1 and RyhB-2, both contributing to attenuated intracellular bacterial growth (Ortega et al., 2012). In Campylobacter jejuni, a fur mutant is significantly affected in its ability to colonize the gastrointestinal tract of chicks (Palyada et al., 2004). As well, a fur mutant of S. enterica 
Table 1

Regulation of virulence and virulence determinants by Fur and RyhB in selected veterinary pathogens.

\begin{tabular}{|c|c|c|c|c|c|}
\hline Natural host & Bacterial species & Regulator & $\begin{array}{l}\text { Animal models } \\
\text { that require } \\
\text { Fur/RyhB for } \\
\text { virulence }\end{array}$ & $\begin{array}{l}\text { Virulence determinants } \\
\text { regulated by Fur/RyhB }\end{array}$ & References \\
\hline \multirow[t]{8}{*}{ Fish } & $\begin{array}{l}\text { Aeromonas salmonicida } \\
\text { subsp. salmonicida }\end{array}$ & Fur & Salmon & Acid resistance & Ebanks et al. (2013) \\
\hline & Pseudomonas fluorescens & Fur & $\begin{array}{l}\text { Japanese } \\
\text { flounder }\end{array}$ & Serum resistance & Wang et al. (2009) \\
\hline & Edwardsiella ictaluri & Fur & $\begin{array}{l}\text { Zebrafish } \\
\text { and catfish }\end{array}$ & Haem acquisition & Santander et al. (2012) \\
\hline & Edwardsiella tarda & Fur & $\begin{array}{l}\text { Japanese } \\
\text { flounder }\end{array}$ & $\begin{array}{l}\text { Invasion of eukaryotic } \\
\text { cells, haemolysin } \\
\text { synthesis }\end{array}$ & $\begin{array}{l}\text { Chakraborty et al. (2011), } \\
\text { Wang et al. (2008) }\end{array}$ \\
\hline & \multirow[t]{2}{*}{ Vibrio vulnificus } & Fur & ND & $\begin{array}{l}\text { Iron acquisition, toxin and } \\
\text { haemolysin synthesis }\end{array}$ & $\begin{array}{l}\text { Kim et al. (2013), Wen } \\
\text { et al. (2012) }\end{array}$ \\
\hline & & RyhB & Mice & ND & Alice et al. (2008) \\
\hline & Vibrio anguillarum & Fur & ND & Haem and iron acquisition & $\begin{array}{l}\text { Balado et al. (2008), } \\
\text { Lemos and Osorio (2007) }\end{array}$ \\
\hline & Vibrio parahaemolyticus & RyhB & ND & Iron acquisition & Tanabe et al. (2013) \\
\hline Swine & $\begin{array}{l}\text { Actinobacillus } \\
\text { pleuropneumoniae }\end{array}$ & Fur & Swine & $\begin{array}{l}\text { Iron acquisition, toxin } \\
\text { synthesis }\end{array}$ & Jacobsen et al. (2005) \\
\hline \multirow[t]{2}{*}{$\begin{array}{l}\text { Domestic and } \\
\text { wild animals } \\
\text { (swine, poultry, } \\
\text { cattle...) }\end{array}$} & $\begin{array}{l}\text { Salmonella enterica } \\
\text { serovar Typhimurium }\end{array}$ & Fur & Mice & $\begin{array}{l}\text { Acid resistance, oxidative } \\
\text { and nitrosative stress } \\
\text { resistance, invasion of and } \\
\text { survival in eukaryotic } \\
\text { cells }\end{array}$ & $\begin{array}{l}\text { Choi et al. (2014), } \\
\text { Ellermeier and Slauch } \\
\text { (2008), Husain et al. } \\
\text { (2014), Troxell et al. } \\
\text { (2011) }\end{array}$ \\
\hline & & RyhB-1,2 & ND & $\begin{array}{l}\text { Acid resistance, oxidative } \\
\text { and nitrosative stress } \\
\text { resistance, motility }\end{array}$ & $\begin{array}{l}\text { Calderon et al. (2014a), } \\
\text { Calderon et al. (2014b), } \\
\text { Kim and Kwon (2013a, } \\
\text { 2013b), Ortega et al. } \\
\text { (2012) }\end{array}$ \\
\hline \multirow[t]{3}{*}{ Poultry } & Campylobacter jejuni & Fur & Chicks & $\begin{array}{l}\text { Iron acquisition, flagellar } \\
\text { biosynthesis }\end{array}$ & $\begin{array}{l}\text { Butcher et al. (2012), } \\
\text { Palyada et al. (2004) }\end{array}$ \\
\hline & $\begin{array}{l}\text { Salmonella enterica } \\
\text { serovar Gallinarum }\end{array}$ & Fur & Chicks & ND & Laniewski et al. (2014) \\
\hline & $\begin{array}{l}\text { Avian pathogenic } \\
\text { Escherichia coli }\end{array}$ & Fur & None & Acid and serum resistance & $\begin{array}{l}\text { Huja et al. (2014), Zhu } \\
\text { et al. (2002) }\end{array}$ \\
\hline
\end{tabular}

ND, not determined.

serovar Gallinarum, the causative agent of fowl typhoid, is fully attenuated in 5-day-old chicks, with a $\mathrm{LD}_{50}$ about 2000-fold higher than that of the wild-type strain (Laniewski et al., 2014). By contrast, the fur mutant of an avian pathogenic $E$. coli strain is as virulent as the wildtype strain when tested by subcutaneous inoculation in a 1-day-old chick model (Zhu et al., 2002).

\subsection{Role of Fur and RyhB in the virulence of human pathogens}

As previously shown with veterinary pathogens, the role of Fur and RyhB in virulence of human bacteria depends on the pathogen. While the presence of these regulators is essential in some infectious models, it is not required in others. In Helicobacter pylori, several studies demonstrated that a fur mutant presents severe stomach colonization defects compared to the wild-type strain in both mice and gerbil models (Pich and Merrell, 2013). Fur is also essential for nontypeable Haemophilus influenzae persistence in a chinchilla model of human otitis media (Harrison et al., 2013). Staphylococcus aureus strains lacking fur exhibit alterations in exoprotein production that correlate with increased susceptibility to neutrophils. Moreover, in a murine model of S. aureus pneumonia, $41 \%$ more animals infected with the wild-type strain succumb to infection when compared to the fur mutant (Torres et al., 2010). By contrast, in a murine model of urinary tract infection, deletion of fur in the uropathogenic E. coli strain CFT073 does not attenuate virulence, whereas a ryhB mutant and a double ryhB fur mutant show significantly reduced bladder colonization (Porcheron et al., 2014). In $V$. cholerae, a fur ryhB double mutant competed poorly with the wild-type strain in a mouse model. This defect is not due to the $r y h B$ mutation, since it was complemented by addition of the fur gene only, and because the ryhB mutant competed well with the wild-type strain (Mey et al., 2005a, 2005b). Both copies of the RyhB transcripts are upregulated in lungs of mice infected with Y. pestis, but the ryhB mutant shows no visible effects on virulence in either subcutaneous or intranasal inoculation models (Deng et al., 2012). In P. aeruginosa, analysis of gene expression in the lungs of various cystic fibrosis patients demonstrated that PrrF sRNAs are consistently expressed in the cystic fibrosis lung environment, suggesting a role of these sRNAs during cystic fibrosis lung infection (Nguyen et al., 2014). Moreover, in a murine model of acute lung infection, all mice infected with a prrF1,2 mutant survive during the entire 28-day course of the experiment, whereas all mice 
infected with either the wild-type strain or the complemented strain succumb to lung infection (Reinhart et al., 2015).

For some pathogens that are human host specific, animal models have not been used to test whether Fur and RyhB are important for virulence. However, studies have been performed using cellular models that mimic in vivo conditions. In S. enterica serovar Typhi, Fur contributes to invasion of epithelial cells and is involved in phagocytosis and intracellular survival in human macrophages. The sRNAs RyhB-1 and RyhB-2 are not required for interaction with epithelial cells, but both sRNAs are important for optimal intracellular replication in macrophages (Leclerc et al., 2013). In a plaque assay, a fur mutant of $S$. dysenteriae produces approximately 10 -fold fewer plaques than the wild-type strain. However, these few plaques are the same size as the wild-type plaques, suggesting a defect in invasion rather than a defect in intracellular multiplication or spread. A fur ryhB double mutant is as invasive and has the same plaque forming efficiency as the wild-type. RyhB thus suppresses plaque formation by inhibiting invasion of eukaryotic cells (Broach et al., 2012; Murphy and Payne, 2007).

Since pathogens colonize different niches within the host during the infection process, they encounter different iron availabilities, explaining why Fur and RyhB can be differentially involved in pathogenesis. However, even if these regulators are not always required for virulence of a bacterial pathogen, they can nevertheless contribute to survival during the colonization process or in the environment. One important question that remains unclear is how these regulators are really involved in virulence. Indeed, Fur and/or RyhB mutants undergo important perturbations in iron homeostasis within the bacterial cell. We can thus assume that these disturbances, depending on their importance, can cause affect bacterial metabolism, thus leading to attenuated strains. Moreover, Fur and RyhB are both involved in the regulation, directly or indirectly, of many virulence determinants that contribute to virulence.

\section{Virulence determinants regulated by Fur and RyhB}

The infectious process of bacterial pathogens requires the expression of an arsenal of virulence factors that allow bacteria to invade eukaryotic cells, survive within cells, acquire metals or resist environmental stresses encountered within the host. Here we review several virulence determinants that Fur and RyhB are known to regulate in response to iron availability (Fig. 2). By virulence determinants, we include all those encoded by true virulence genes, virulence-associated genes and virulence life-style genes, as defined by Wassenaar and Gaastra (2001).

\subsection{Iron and haem acquisition}

Due to the biological importance of iron, many iron and haem uptake systems are involved in bacterial pathogenicity. As the master iron homeostasis regulator, iron and haem acquisition systems are directly repressed by Fur in iron-replete conditions in both Gram-negative and Grampositive bacteria (Harrison et al., 2013; Helmann, 2014; Holmes et al., 2005; Leclerc et al., 2013; Lemos and Osorio, 2007; Mey et al., 2005b; Palyada et al., 2004; Payne et al., 2006; Porcheron et al., 2013, 2014; Santander et al., 2012; Torres et al., 2010). Fur can also indirectly regulate iron acquisition. Indeed, in $P$. aeruginosa, Fur represses expression of $p v d S$, encoding an extracytoplasmic function sigma factor that directly activates expression of genes for pyoverdin siderophore biosynthesis and uptake (Ochsner et al., 1996).

RyhB regulation of iron acquisition and homeostasis is well established. The first description of its role in siderophore production was in a non-pathogenic $E$. coli $\mathrm{K}-12$ strain. In this strain, RyhB promotes synthesis of the siderophore enterobactin by repressing the translation of cysE mRNA, which allows more serine to be used for enterobactin production, and by activating shiA mRNA translation to increase shikimate (a metabolic precursor of enterobactin biosynthesis) acquisition from the environment. RyhB is also required for normal expression of the polycistronic enterobactin biosynthesis operon, entCEBAH (Salvail and Massé, 2012). Several recent studies also describe a role for this sRNA in regulating iron acquisition in pathogenic bacteria. In the uropathogenic $E$. coli strain CFT073, a ryhB mutant produces less of the three types of siderophores produced by this strain (enterobactin, salmochelins and aerobactin), as RyhB activates several genes involved in siderophore biosynthesis such as shiA and $i u c D$, the latter being involved in aerobactin biosynthesis. This regulation of iucD can also explain the role of RyhB in bladder colonization as this gene is downregulated in bladders infected by a ryhB mutant compared to bladders infected with the wild-type strain (Porcheron et al., 2014). In $V$. parahaemolyticus, it has been shown that RyhB confers increased stability of the polycistronic mRNA involved in vibrioferrin siderophore biosynthesis (Tanabe et al., 2013). In $V$. cholerae, microarray analysis demonstrated that expression of several genes involved in iron acquisition such as fhuAC, feoAB and irgA is increased by RyhB (Mey et al., 2005a). In K. pneumoniae, the additional deletion of ryhB in a fur mutant reduces the expression of several genes (iucA, fepAB, entC, fec $A E$ ) corresponding to three iron acquisition systems in K. pneumoniae (aerobactin, enterobactin and ferric citrate), and results in reduced siderophore production (Huang et al., 2012). By contrast, a $P$. aeruginosa prrF1,2 mutant of strain PAO1 is not defective for siderophore production, implying that virulence attenuation of this mutant is not linked to a defect in iron acquisition. Moreover, expression of phuS, which controls the flux of haem, is significantly increased in the prrF1,2 mutant compared to the wild-type strain. Expression of phuS occurs via the PrrH sRNA, whose expression is dependent on arrangement of the prrF1 and prrF2 genes (Reinhart et al., 2015).

\subsection{Invasion of and survival in eukaryotic cells}

During the course of infection, $S$. Typhimurium invades non-phagocytic epithelial cells in the small intestine via a type III secretion system (T3SS) encoded on Salmonella 


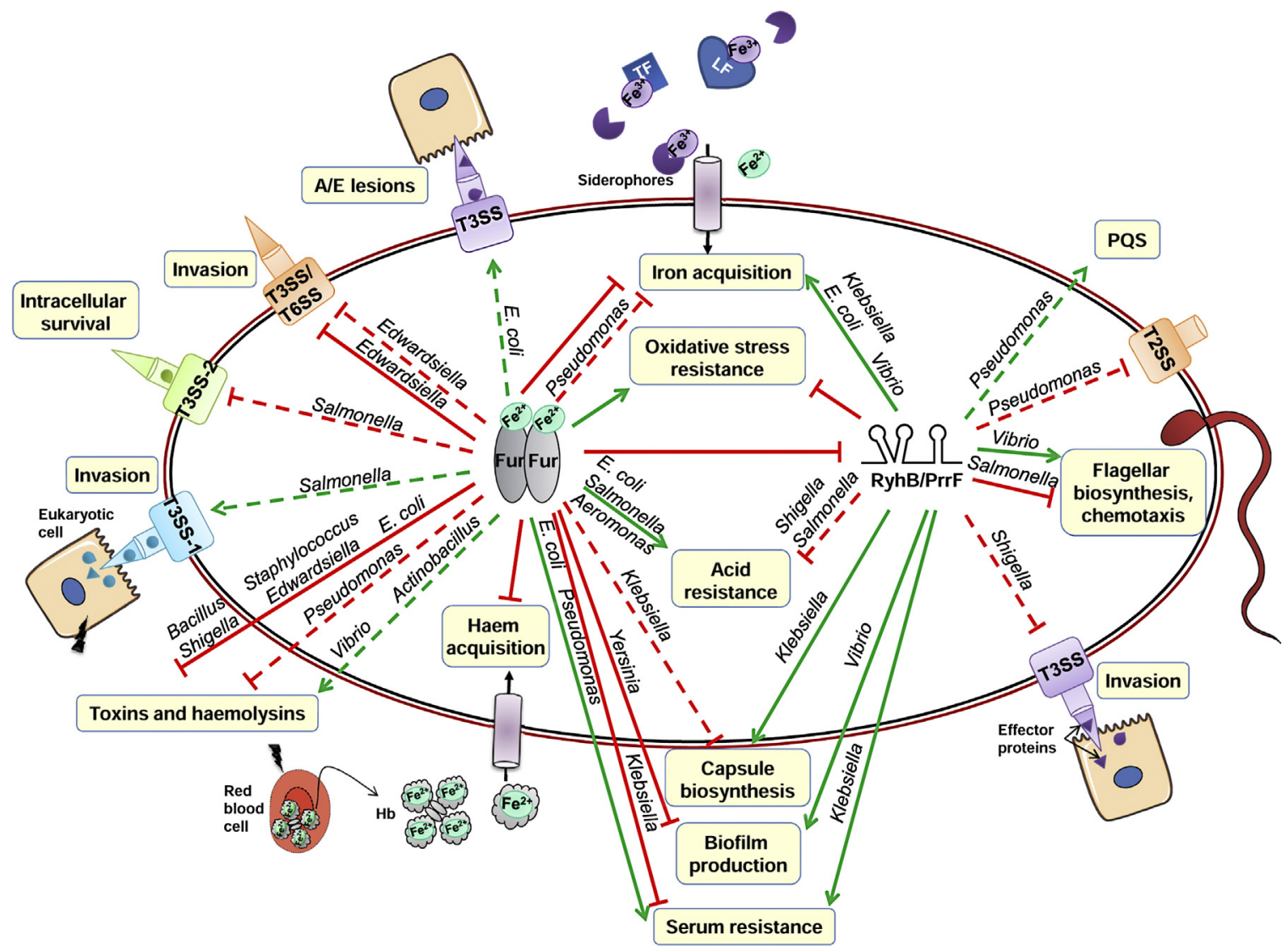

Fig. 2. Virulence determinants regulated by Fur and RyhB in pathogenic bacteria. Fur (in iron-rich environments) and RyhB (in iron-poor environments) can regulate either directly (solid lines) or indirectly (dotted lines), via other regulators or intracellular iron concentration modulation, the expression of many genes involved in production of virulence determinants. Virulence phenotypes can be either activated (green lines) or repressed (red lines) by Fur or RyhB. Bacterial genera in which a phenotype has been described are indicated. Bacterial genera are not indicated when a phenotype is common to all pathogens. T2SS, type-two secretion system; T3SS, type-three secretion system; T6SS, type-six secretion system; PQS, Pseudomonas quinolone signal; A/E, attachment/ effacement; TF, transferrin; LF, lactoferrin; Hb, haemoglobin. (For interpretation of the references to colour in this figure legend, the reader is referred to the web version of the article.)

pathogenicity island 1 (SPI-1). The SPI-1 T3SS (T3SS-1) forms a needle-like complex that is responsible for the direct injection of bacterial effector proteins into the host cell cytosol. HilA is the central activator of the SPI-1 T3SS, itself being regulated by three transcriptional activators: HilC, HilD and RtsA. It has been shown that Fur was able to induce hilA expression, this regulation being mediated through HilD and H-NS. Indeed, Fur represses hns expression, H-NS being itself involved in hilA repression, possibly through the repression of hilD (Ellermeier and Slauch, 2008; Troxell et al., 2011) (Fig. 3). Once inside the host cell, $S$. Typhimurium survives and proliferates inside membrane-bound vacuoles. As a means for survival, Salmonella employs a series of proteins produced from the Salmonella pathogenicity island 2 (SPI-2). SPI-2 encodes a T3SS (T3SS-2) apparatus and its substrate effector proteins. Once produced within the phagosome, the effector proteins are delivered into the macrophage cytoplasm via the T3SS-2 apparatus, where these proteins interfere with host bacterium-killing processes. The SsrA/B two-component system promotes the expression of SPI-2 genes inside macrophages and under acidic conditions. Fur controls SPI-2 expression by directly repressing $s s r B$ expression (Choi et al., 2014) (Fig. 3). The regulation of SPI-1 and SPI-2 genes expression could explain the important role of Fur in the virulence of $S$. Typhimurium (Troxell et al., 2011).

After ingestion, Shigella transits the acidic environment of the stomach then initiates disease by invading colonic epithelial cells, multiplying intracellularly, and spreading to adjacent epithelial cells via actin-based motility (Payne et al., 2006). Shigella virulence therefore is dependent on the capacity to invade epithelial cells, survive intracellularly and spread to adjacent cells. These virulence traits require many actors, such as T3SS and its effector proteins to invade. The coordinated regulation of expression of these virulence-associated determinants is largely mediated by the activity of two transcriptional activators, VirF and VirB. VirF directly activates the transcription of twovirulence associated genes, ics $A$ and virB. IcsA facilitates intracellular spread by mediating actin-based motility, while VirB directly promotes the expression of multiple 


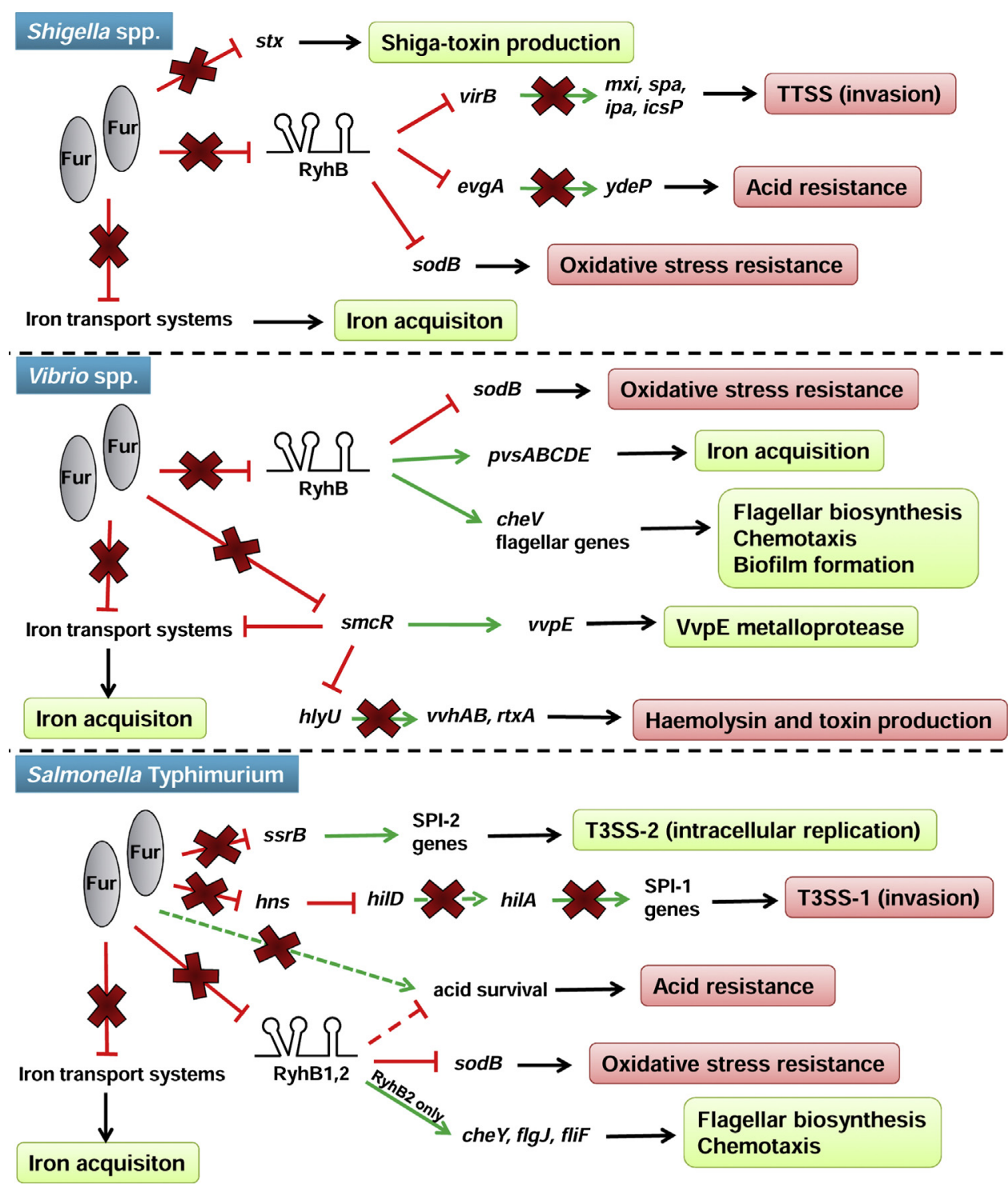

Fig. 3. Details of some Fur/RyhB regulatory cascades mediating virulence determinant regulation in low-iron environments. In iron-poor environments, such as the intracellular cytoplasmic or lysosomal environment, Fur activity is inhibited, reducing its regulatory function. Target genes are thus activated or repressed, and RyhB-dependent regulation can occur. Virulence determinants that are thus activated following Fur inactivation are indicated in green, and those that are inhibited are indicated in red. (For interpretation of the references to colour in this figure legend, the reader is referred to the web version of the article.)

virulence-associated genes such as genes encoding components of the T3SS (spa, ipa), icsP encoding the protease that modulates IcsA activity, and mxiE encoding a transcriptional activator of additional virulence associated genes. It has been shown that RyhB represses virB gene expression, independently of virF (Fig. 3). This allows reduced production of the T3SS while simultaneously allowing intracellular spreading via IcsA. This explains how RyhB suppresses plaque formation by inhibiting invasion of eukaryotic cells (Broach et al., 2012; Murphy and Payne, 2007).

In E. tarda, type III and VI secretion systems (T3SS and T6SS) are determined as the two most important virulence mechanisms. Fur represses directly evpP in the T6SS gene cluster. Moreover, Fur indirectly regulates esrC, encoding a regulator of T3SS and T6SS, thus leading to a decrease in production of T3SS and T6SS. In E. tarda, Fur interacts directly with EsrC protein and indirectly represses esrC expression (Chakraborty et al., 2011).

In P. aeruginosa strain PAO1, expression of the vreR gene encoding an anti-sigma factor of the Vre extracytoplasmic function cell surface signalling system is upregulated in a prrF1,2 mutant. VreR regulates expression of the Hxc type II secretion system, involved in the secretion of alkaline phosphatase, and of a putative two-partner secretion system (Reinhart et al., 2015).

Enterohemorrhagic and enteropathogenic $E$. coli cause lesions known as attaching and effacing $(\mathrm{A} / \mathrm{E})$ lesions, 
characterized by intimate attachment of the bacteria to the enterocyte membrane, destruction of the brush border microvilli and striking cytoskeletal changes that include accumulation of polymerized actin beneath the site of attachment, forming pedestal-like structures. The intimate attachment to the enterocyte surface is mediated through interactions of the bacterial outer membrane protein intimin and the translocated intimin receptor (Tir). Tir and other effector proteins are delivered to the host cell via a T3SS. The virulence factors necessary for A/E lesion induction are primarily encoded by the LEE (locus of enterocyte effacement) region. The LEE also encodes the transcriptional regulator Ler, which activates the transcription of other LEE genes. It has recently been reported that the loss of Fur affects the translation of the ler gene by increasing the intracellular concentration of iron, this regulation being mediated by the antisense RNA arl. Interference with the translation of ler could be mediated by a ler mRNA-antisense RNA complex, which is accelerated by a high concentration of free iron, or superoxide and hydroxyl radicals produced by free iron. The authors thus propose a novel mechanism for ler expression in which the expression of this gene is regulated at the level of translation in response to the iron concentration through interference by a transcript from the antisense strand (Tobe et al., 2014). As this mechanism is mediated by iron concentration rather than Fur itself, it remains to be determined if arl expression is under the control of another transcriptional regulator, itself being regulated directly by Fur.

\subsection{Motility, chemotaxis and biofilm formation}

The ability of $V$. cholerae to form biofilms in aquatic environments is critical for its persistence in these environments and for efficient spread to the human host, thus biofilms are an important aspect of the pathogenic potential of this organism. Several genes involved in flagellar biosynthesis or chemotaxis are downregulated in a $V$. cholerae ryhB mutant. RyhB is thus important for biofilm formation and chemotactic motility, even if not required for colonization in a mouse model (Mey et al., 2005a). By contrast, in S. Typhimurium, RyhB2 is involved in the downregulation of the flagellar and chemotaxis genes $\mathrm{flgJ}$, cheY and fliF, thus leading to an increased motility phenotype in a ryhB2 mutant strain (Kim and Kwon, 2013b) (Fig. 3). In Y. pestis, Fur inhibits biofilm formation by directly repressing $h m s T$ expression, thus leading to inhibition of c-di-GMP synthesis (Sun et al., 2012).

\subsection{Capsule biosynthesis}

In K. pneumoniae, capsular polysaccharide (CPS) is a major determinant of pathogenesis that protects bacteria from phagocytosis and killing by serum factors. It also promotes bacterial colonization and biofilm formation at the infection sites. The $\mathrm{K} 2 \mathrm{cps}$ gene cluster transcription is controlled by several transcription factors such as the Rcs system, RmpA or RmpA2. Fur represses the expression of rcsA, rmpA and rmpA2 to decrease CPS biosynthesis. Moreover, RyhB increases the transcriptional level of the
K2 cps gene cluster to increase CPS biosynthesis, without affecting the transcription level of $\operatorname{rcs} A, \operatorname{rmpA}$ and $r m p A 2$ (Huang et al., 2012).

\subsection{Toxin and haemolysin synthesis}

In Bacillus cereus, haemolysin II (HlyII) is one of the secreted factors responsible for causing haemolysis and is found with increased incidence in pathogenic strains. The toxic properties of HlyII rely on its ability to disrupt cellular and artificial membranes by pore formation. It has been shown that Fur is able to repress directly hlyII expression, by obstructing the binding of RNA polymerase to the hlyII promoter (Sineva et al., 2012). In E. tarda, Fur represses eth $B$ which encodes the activation/secretion apparatus of the haemolysin system (Eth) (Chakraborty et al., 2011). In EHEC and S. dysenteriae, stx genes encoding Shiga-toxin are directly repressed by Fur (Payne et al., 2006; Tobe et al., 2014). In S. aureus, a fur mutant exhibits increased haemolysis on blood agar plates compared to the wildtype strain. This phenotype depends on the regulation of the alpha-toxin encoding gene hla. Moreover, fur inactivation increases the cytotoxic potential of $S$. aureus with different human epithelial cell lines and with human promyelocytic cells (Torres et al., 2010). One virulence factor of $P$. aeruginosa is the exotoxin $A$, encoding by the toxA gene. Expression of toxA is activated by the PvdS sigma factor, itself being repressed by Fur (Ochsner et al., 1996). Whereas Fur represses toxin or haemolysin production in some bacterial species, it is also able to activate this production in other species. For instance, the expression of apxI, encoding one of the RTX toxins that are major virulence factors of $A$. pleuropneumanie, is positively regulated by Fur under high calcium conditions (Jacobsen et al., 2005). In V. vulnificus, Fur directly inhibits expression of the smcR gene, encoding the master regulator of quorum sensing. SmcR induces the expression of $v v p E$, a gene encoding an elastase, and represses yeg $D$, a gene encoding a chaperone. SmcR also represses the expression of $h l y U$, a gene encoding an activator that induces the expression of the virulence factor genes $v v h A B$ and $r t x A$, encoding a haemolysin and a RTX toxin, respectively. By inhibiting smcR expression, Fur indirectly activates toxin production (Kim et al., 2013) (Fig. 3).

\subsection{Quorum sensing}

In $P$. aeruginosa, the expression of many virulence factors is controlled by signalling molecules that are synthesized and secreted by this bacterium. One of these signalling systems is the Pseudomonas quinolone signal (PQS) quorum sensing system which involves anthranilate as a precursor. The PrrF sRNAs are involved in the repression of anthranilate degradation by repressing ant $A B C$, thus allowing for PQS biosynthesis (Oglesby et al., 2008).

\subsection{Serum resistance}

While most bacteria are unable to endure the strong bactericidal effect of serum, several pathogens have 
evolved mechanisms that enable them to subvert the host defense systems and successfully survive in serum. Surface structures such as lipopolysaccharide and polysaccharide capsule can inhibit complement-dependent killing. The role of Fur and RyhB in this resistance seems to differ depending on the pathogen. For instance, a fur mutant of $P$. fluorescens exhibits decreased resistance against host serum bactericidal activity (Wang et al., 2009), whereas a K. pneumoniae fur mutant exhibits higher resistance to serum than the wild-type strain. Moreover, additional ryhB mutation in the fur mutant results in restoration of serum resistance to the wild-type level. The protective role of RyhB against serum killing could be due to the activation of CPS biosynthesis (Huang et al., 2012). In an avian pathogenic E. coli strain, Fur is responsible for more than $80 \%$ of human serum-induced transcriptional response and a fur mutant is completely serum sensitive (Huja et al., 2014). As a fur mutant of another avian pathogenic $E$. coli strain has been shown to be as virulent as the wild-type strain in 1-day-old chickens (Zhu et al., 2002), we can hypothesize that the immune system of 1-day-old-chicken, particularly the complement system, is not fully developed. It could be thus interesting to test the effect of a fur mutation in an avian pathogenic $E$. coli strain with older chickens as in the colibacillosis or air sac infection model in chickens.

\subsection{Acid resistance}

During infection, pathogenic bacteria encounter various low-pH environments in which they have to survive. For instance, there are two stages during the process of infection in which acid resistance would be required for successful pathogenesis of $S$. Typhimurium. One is the gastric acidity in the stomach that Salmonella ingested with contaminated food materials would pass through, and the other one is the acidification of the phagolysosome which Salmonella would encounter when phagocytosed by macrophages. In S. Typhimurium, the acid survival of the fur mutant is dramatically reduced compared to the wild-type strain. Acid resistance is restored slightly by ryhB-1 deletion in the fur mutant, but substantially by a ryhB-2 deletion. In a triple deletion mutant ( fur, ryhB-1 and $r y h B-2)$, there is an additive increase in acid resistance by the deletion in ryhB-1 and $r y h B-2$. Moreover, the acid resistance of the triple mutant is still significantly lower than that of the wild-type strain, indicating the presence of RyhB-independent, Fur-regulated genes that are important in acid survival (Kim and Kwon, 2013a). The same acid sensitivity phenotype is observed for fur mutants of an avian pathogenic E. coli strain and A. salmonicida, but the role of RyhB has not been investigated in these studies (Ebanks et al., 2013; Zhu et al., 2002).

Shigella can resist the acidic conditions of the stomach via two acid resistance systems, a glutamate-dependant and a glutamate-independent system. Both systems are regulated by the EvgA response regulator, which promotes $y d e P$ expression, a gene encoding an oxidoreductase involved in acid resistance. In $S$. flexneri, RyhB allows indirect repression of $y d e P$ via repression of $\operatorname{evg} A$, thus resulting in acid sensitivity phenotype (Oglesby et al., 2005) (Fig. 3).

\subsection{Oxidative and nitrosative stress resistance}

During infection of the host, a cascade of anti-bacterial processes is initiated by professional phagocytes, including the production of nitric oxide (NO) or reactive oxygen species (ROS). These agents exert powerful antimicrobial effects by reacting with molecular targets such as transition metals, thiols, lipids and DNA. For bacterial pathogens, effective detoxification systems for antimicrobial reactive oxygen and nitrogen species are important to establish a successful infection and to survive inside the host (Fang, 2004). Within macrophages, Salmonella is sequentially exposed to the antimicrobial activity of the NADPH oxidase and inducible nitric oxide synthase flavohemoproteins. Following the initial oxidative burst emanating from the NADPH oxidase, reactive nitrogenspecies derived iNOS (inducible nitric oxide synthase)expressing human and murine macrophages exert sustained bacteriostatic activity at later stages of the intracellular Salmonella infection (Husain et al., 2014). In $S$. Typhimurium, a fur mutant is hypersusceptible to nitric oxide. Indeed, the reduced cytochrome content of the fur mutant decreases antinitrosative defenses. Fur contributes to virulence of the strain by protecting against NO-dependent host defenses, as addition of an iNOS-specific inhibitor in the drinking water of mice significantly increased the virulence of a fur mutant. However the role that RyhB-1 or RyhB- 2 could play in this mutant has not been investigated (Husain et al., 2014). In another study performed in S. Typhimurium, RyhB-1 and RyhB-2 are induced under nitrosative stress conditions, downregulated the expression of cytochrome oxidase complexes and upregulated the expression of the nitrite reductase system to respond to GSNO ( $S$-nitrosoglutathione) stress (Calderon et al., 2014b).

Oxidative stress is another challenge that bacteria have to overcome to establish infection in mammalian hosts. Indeed, mammals use $\mathrm{H}_{2} \mathrm{O}_{2}$ to create an oxidative environment to protect themselves against pathogens. Moreover, the presence of free intracellular iron aggravates oxidative stress in bacteria via the Fenton reaction by producing ROS, a reaction that is mitigated by cytoplasmic proteins that scavenge free iron such as Dps and ferritins [for a review concerning oxidative stress and iron starvation, see (Benjamin et al., 2010)]. In many bacterial pathogens, the defense against ROS requires Fur. Several transcriptional analyses studies have demonstrated that many genes implicated in oxidative stress resistance such as sod, suf and dps genes are regulated by Fur (Holmes et al., 2005; Mey et al., 2005b; Oglesby et al., 2005). However, the potential role of RyhB in this regulation has not been determined. Many recent studies tend to demonstrate that RyhB could be also implicated in oxidative stress resistance. In $S$. Typhi, a fur mutant is sensitive to oxidative stress, the expression of sRNAs RyhB-1 and 2 being responsible for this phenotype. Indeed, the strains harbouring deletion of fur and one of the sRNAs are also significantly more sensitive than the wild-type strain, but are less sensitive than the fur mutant. When both sRNAs are deleted in the fur mutant, oxidative stress survival is restored to the wild type level (Leclerc et al., 2013). In a 
similar way, the survival of a fur mutant of $S$. Typhimurium is significantly decreased as compared to the wild-type strain in the presence of hydrogen peroxide. This reduced survival is then increased by deleting $r y h B-1$ or $r y h B-2$. However, the triple mutant is still more sensitive than the wild-type strain, indicating the presence of $r y h B$-independent, Fur-regulated genes important in oxidative stress resistance (Kim and Kwon, 2013a). Moreover both RyhB-1 and RyhB-2 are induced by OxyR in response to oxidative stress (Calderon et al., 2014a). In the uropathogenic E. coli strain CFT073, a fur mutant is also significantly more sensitive to oxidative stress than the wild-type strain. The additional deletion of $r y h B$ in the fur mutant does not have an impact on survival, indicating that only Fur (and not RyhB) is important in oxidative stress resistance (Porcheron et al., 2014). Finally, depending on the strain, the role of RyhB and Fur in oxidative stress resistance differs and more studies would be useful to decrypt the molecular mechanisms involved between iron homeostasis and oxidative stress resistance.

ROS are produced as a consequence of aerobic metabolism. In anaerobic environments, soluble iron is abundant and the lack of oxygen precludes the formation of deleterious ROS. Therefore, the need for sophisticated mechanisms of an iron-dependent response is questionable. Whereas Fur seems to be of limited importance for virulence of some anaerobic bacteria such as Dichelobacter nodosus or Desulfovibrio vulgaris, it plays a crucial role in other anaerobic bacteria. Indeed, a fur mutant of the strictly anaerobic bacterium Clostridium acetobutylicum is also more sensitive to $\mathrm{H}_{2} \mathrm{O}_{2}$ and $\mathrm{O}_{2}$ oxidative stress compared to the wild-type strain, demonstrating the importance of Fur for maintaining the intracellular iron balance even in some anaerobic strains (Vasileva et al., 2012).

\section{Fur and RyhB modes of action: from direct to indirect regulation of virulence determinants}

Based on the studies described above, we can highlight three modes of action of Fur/RyhB regulation (Fig. 4). Firstly, Fur and RyhB can act directly to regulate the expression of a gene. This is especially the case for genes involved in iron acquisition, as they are directly repressed by Fur. The direct action of RyhB on the expression of genes involved in siderophore production in $V$. parahaemolyticus has also been demonstrated (Tanabe et al., 2013). This mode of action allows regulators to fine tune the expression of a specific target, to ensure a specific response to a specific environment. In these cases, Fur and RyhB allow a targeted response to iron availability. However, even if the direct regulation of some genes by Fur/RyhB has been demonstrated, further investigations may identify other yet unknown factors within the regulatory cascade allowing the expression of these virulence phenotypes. For instance, no other factors between RyhB and flagellar genes are currently known to be involved in the regulation of the motility phenotype in $V$. cholerae or $S$. Typhimurium, but future studies could identify a RyhB-dependent transcriptional factor that could directly regulate expression of these genes.

The second mode of action of Fur/RyhB regulation involves other transcriptional regulators. For example, Fur and RyhB target transcriptional regulators, such as VirB in S. dysenteriae. This allows bacteria, when they are in the intracellular environment containing low iron, to inhibit components and effector proteins of the T3SS at the same time. Indeed, once within the intracellular environment, Shigella must repress production of the T3SS in order to prevent premature lysis of the eukaryotic cell. This implies that all genes involved in this phenotype, i.e. T3SS

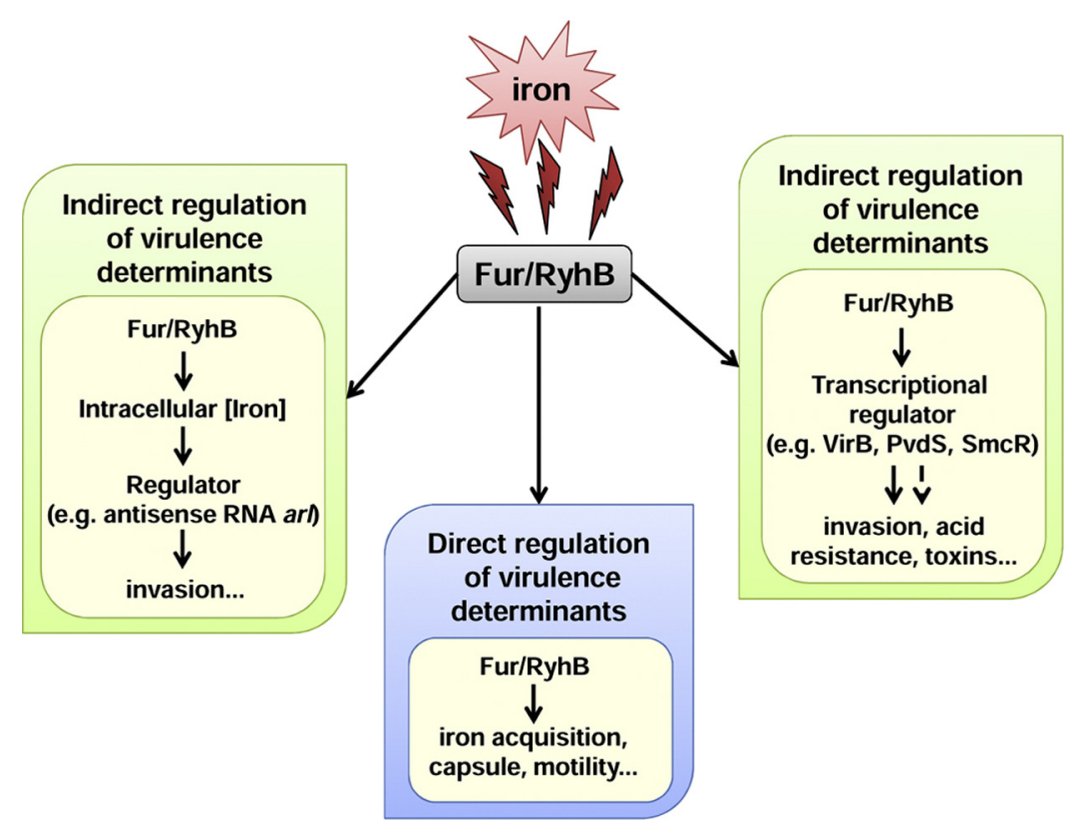

Fig. 4. Mechanisms of direct and indirect regulation of virulence determinants by Fur and RyhB in response to environmental iron concentration. 
components and effector proteins, are regulated at a same time, a role perfectly fulfilled by Fur/RyhB regulation in response to iron availability (Broach et al., 2012; Murphy and Payne, 2007). In S. Typhimurium, Fur regulation of the transcriptional regulators $\mathrm{H}-\mathrm{NS}$ and SsrB allows bacteria to express either the T3SS-1 or the T3SS-2 depending on iron availability (Choi et al., 2014; Ellermeier and Slauch, 2008; Troxell et al., 2011) (Fig. 3). This depends thus on the presence of the bacteria either in the extracellular environment (that requires T3SS-1 expression for invasion of eukaryotic cells) or in the intracellular environment (that requires T3SS-2 to survive and proliferate). By regulating transcriptional factors, the impact of RyhB/ Fur regulation is thus more important and can differentially modulate expression of genes that may be advantageous or deleterious under specific conditions that can be achieved through responses to level of iron availability.

Finally, the last mode of action of Fur/RyhB regulation has been described recently, with the regulation of LEE genes in E. coli. This regulation occurs via the Ler transcriptional regulator but neither Fur nor RyhB regulates directly the expression of this regulator. By regulating iron homeostasis, Fur and RyhB modulate the intracellular concentration of iron, and this concentration allows regulation of ler expression via an antisense RNA (Tobe et al., 2014). Contrary to the previous mode of action where Fur or RyhB directly regulate a transcriptional factor that controls virulence determinants, in the latter example Fur indirectly regulates the antisense RNA that controls expression of virulence determinants. It would not be surprising if future studies describe new RyhB/Fur indirect targets responding also to iron concentration rather than direct action of Fur or RyhB. Once again, this mode of action would have a large impact by regulating several different networks of genes involved in virulence or environmental adaptation through simultaneous regulation of transcriptional regulators including non-coding RNAs and regulatory proteins.

\section{Concluding remarks}

The reports presented in this review have made significant contributions to further elucidate the role of Fur and RyhB in pathogenesis through regulation of virulence determinants in a broad range of bacterial species. Whereas in some species such as Shigella spp. mechanisms mediating regulation of virulence determinants by Fur and RyhB are more fully established, further studies will be necessary to evaluate and to understand the global impact of these major regulators in virulenceassociated traits of other pathogenic bacteria. It is now evident that iron is not only an essential metal for biological functions in microorganisms, but it is also an environmental signal and a regulatory element that allows bacteria to respond rapidly and efficiently to iron starvation encountered within the host by expressing or repressing multiple genes encoding for proteins important for adaptive responses and virulence. By responding to iron availability in its environment, the pathogen thus favours its chances to survive, replicate and cause disease within the host. As they regulate many virulence determinants in a variety of pathogens, fur and ryhB mutants should now be considered as potential strategic tools in the fight against pathogenic bacteria. Indeed, by targeting these transcriptional regulators, a number of virulence determinants responding to iron signalling could also be targeted. Some research is beginning to evaluate the potential of fur mutant strains as vaccines. For instance, a fur mutant of $E$. ictaluri is immune protective in zebrafish and catfish, triggering systemic immunity. A fur mutant could be thus successfully used to attenuate E. ictaluri in order to develop effective live attenuated vaccines for the aquaculture industry (Santander et al., 2012). More recently, a fur attenuation mutant of $S$. Gallinarum elicited excellent protection against lethal $S$. Gallinarum challenge in poultry. This deletion mutant is highly efficacious as a live vaccine and can protect chickens against fowl typhoid when delivered orally or intramuscularly (Laniewski et al., 2014). It is also proposed that a $P$. aeruginosa prrF1,2 mutant could represent a potential candidate for future vaccine development. Indeed, intranasal inoculation of mice with this mutant generated a protective immune response against the wild-type strain (Reinhart et al., 2015). Finally, future studies of Fur/RyhB regulation would undoubtedly yield a more comprehensive understanding of the complex role of iron in both bacterial physiology and pathogenicity. Such research advances could lead to a more targeted approach for the development of future vaccines for the prevention of animal diseases of economic and public health importance.

\section{Conflict of interest statement}

The authors declare that no conflicts of interest exist.

\section{Acknowledgements}

This work was supported by a postdoctoral fellowship from the Fondation Armand-Frappier (G.P.), a team grant from Fonds Québécois de la Recherche sur la Nature et les Technologies (2011 -PR-139139) (C.M.D.), the Natural Sciences and Engineering Research Council Canada (NSERC) Discovery Grants(RGPIN 250129-07 and 201406622) (C.M.D.), the Centre de Recherche en Infectiologie Porcine et Aviaire (2014-RS-170946) (C.M.D.), and the Canada Research Chairs program (950-209182) (C.M.D.).

\section{References}

Alice, A.F., Naka, H., Crosa, J.H., 2008. Global gene expression as a function of the iron status of the bacterial cell: influence of differentially expressed genes in the virulence of the human pathogen Vibrio vulnificus. Infect. Immun. 76, 4019-4037.

Andrews, S.C., Robinson, A.K., Rodriguez-Quinones, F., 2003. Bacterial iron homeostasis. FEMS Microbiol. Rev. 27, 215-237.

Baichoo, N., Helmann, J.D., 2002. Recognition of DNA by Fur: a reinterpretation of the Fur box consensus sequence. J. Bacteriol. 184 5826-5832.

Balado, M., Osorio, C.R., Lemos, M.L., 2008. Biosynthetic and regulatory elements involved in the production of the siderophore vanchrobactin in Vibrio anguillarum. Microbiology 154, 1400-1413.

Benjamin, J.A., Desnoyers, G., Morissette, A., Salvail, H., Masse, E., 2010. Dealing with oxidative stress and iron starvation in microorganisms: an overview. Can. J. Physiol. Pharmacol. 88, 264-272.

Broach, W.H., Egan, N., Wing, H.J., Payne, S.M., Murphy, E.R., 2012. VirFindependent regulation of Shigella virB transcription is mediated by the small RNA RyhB. PLoS ONE 7, e38592. 
Butcher, J., Sarvan, S., Brunzelle, J.S., Couture, J.F., Stintzi, A., 2012. Structure and regulon of Campylobacter jejuni ferric uptake regulator Fur define apo-Fur regulation. Proc. Natl. Acad. Sci. U. S. A. 109, 10047-10052.

Calderon, I.L., Morales, E.H., Collao, B., Calderon, P.F., Chahuan, C.A., Acuna, L.G., Gil, F., Saavedra, C.P., 2014a. Role of Salmonella Typhimurium small RNAs RyhB-1 and RyhB-2 in the oxidative stress response. Res. Microbiol. 165, 30-40.

Calderon, P.F., Morales, E.H., Acuna, L.G., Fuentes, D.N., Gil, F., Porwollik, S., McClelland, M., Saavedra, C.P., Calderon, I.L., 2014b. The small RNA RyhB homologs from Salmonella typhimurium participate in the response to $S$-nitrosoglutathione-induced stress. Biochem. Biophys. Res. Commun. 450, 641-645.

Chakraborty, S., Sivaraman, J., Leung, K.Y., Mok, Y.K., 2011. Two-component PhoB-PhoR regulatory system and ferric uptake regulator sense phosphate and iron to control virulence genes in type III and VI secretion systems of Edwardsiella tarda. J. Biol. Chem. 286, 39417-39430.

Choi, E., Kim, H., Lee, H., Nam, D., Choi, J., Shin, D., 2014. The iron-sensing fur regulator controls expression timing and levels of Salmonella pathogenicity island 2 genes in the course of environmental acidification. Infect. Immun. 82, 2203-2210.

Deng, Z., Meng, X., Su, S., Liu, Z., Ji, X., Zhang, Y., Zhao, X., Wang, X., Yang, R., Han, Y., 2012. Two sRNA RyhB homologs from Yersinia pestis biovar microtus expressed in vivo have differential Hfq-dependent stability. Res. Microbiol. 163, 413-418.

Ebanks, R.O., Goguen, M., Knickle, L., Dacanay, A., Leslie, A., Ross, N.W., Pinto, D.M., 2013. Analysis of a ferric uptake regulator (Fur) knockout mutant in Aeromonas salmonicida subsp. salmonicida. Vet. Microbiol. $162,831-841$

Ellermeier, J.R., Slauch, J.M., 2008. Fur regulates expression of the Salmonella pathogenicity island 1 type III secretion system through HilD. J. Bacteriol. 190, 476-486.

Fang, F.C., 2004. Antimicrobial reactive oxygen and nitrogen species: concepts and controversies. Nat. Rev. Microbiol. 2, 820-832.

Harrison, A., Santana, E.A., Szelestey, B.R., Newsom, D.E., White, P., Mason, K.M., 2013. Ferric uptake regulator and its role in the pathogenesis of nontypeable Haemophilus influenzae. Infect. Immun. 81, 1221-1233.

Helmann, J.D., 2014. Specificity of metal sensing: iron and manganese homeostasis in Bacillus subtilis. J. Biol. Chem. 289, 28112-28120.

Holmes, K., Mulholland, F., Pearson, B.M., Pin, C., McNicholl-Kennedy, J., Ketley, J.M., Wells, J.M., 2005. Campylobacter jejuni gene expression in response to iron limitation and the role of Fur. Microbiology 151, 243-257.

Hood, M.I., Skaar, E.P., 2012. Nutritional immunity: transition metals at the pathogen-host interface. Nat. Rev. Microbiol. 10, 525-537.

Huang, S.H., Wang, C.K., Peng, H.L., Wu, C.C., Chen, Y.T., Hong, Y.M., Lin, C.T., 2012. Role of the small RNA RyhB in the Fur regulon in mediating the capsular polysaccharide biosynthesis and iron acquisition systems in Klebsiella pneumoniae. BMC Microbiol. 12, 148.

Huja, S., Oren, Y., Biran, D., Meyer, S., Dobrindt, U., Bernhard, J., Becher, D., Hecker, M., Sorek, R., Ron, E.Z., 2014. Fur is the master regulator of the extraintestinal pathogenic Escherichia coli response to serum. MBio 5.

Husain, M., Jones-Carson, J., Liu, L., Song, M., Saah, J.R., Troxell, B., Mendoza, M., Hassan, H., Vazquez-Torres, A., 2014. Ferric uptake regulator-dependent antinitrosative defenses in Salmonella enterica serovar Typhimurium pathogenesis. Infect. Immun. 82, 333-340.

Jacobsen, I., Gerstenberger, J., Gruber, A.D., Bosse, J.T., Langford, P.R., Hennig-Pauka, I., Meens, J., Gerlach, G.F., 2005. Deletion of the ferric uptake regulator Fur impairs the in vitro growth and virulence of Actinobacillus pleuropneumoniae. Infect. Immun. 73, 3740-3744.

Kim, I.H., Wen, Y., Son, J.S., Lee, K.H., Kim, K.S., 2013. The fur-iron complex modulates expression of the quorum-sensing master regulator, SmcR, to control expression of virulence factors in Vibrio vulnificus. Infect. Immun. 81, 2888-2898.

Kim, J.N., Kwon, Y.M., 2013a. Genetic and phenotypic characterization of the RyhB regulon in Salmonella Typhimurium. Microbiol. Res. 168, 41-49.

Kim, J.N., Kwon, Y.M., 2013b. Identification of target transcripts regulated by small RNA RyhB homologs in Salmonella: RyhB-2 regulates motility phenotype. Microbiol. Res. 168, 621-629.

Laniewski, P., Mitra, A., Karaca, K., Khan, A., Prasad, R., Curtiss, R.3rd, Roland, K.L., 2014. Evaluation of protective efficacy of live attenuated Salmonella enterica serovar Gallinarum vaccine strains against fowl typhoid in chickens. Clin. Vaccine Immunol. 21, 1267-1276.

Leclerc, J.M., Dozois, C.M., Daigle, F., 2013. Role of the Salmonella enterica serovar Typhi Fur regulator and small RNAs RfrA and RfrB in iron homeostasis and interaction with host cells. Microbiology 159, 591-602.
Lemos, M.L., Osorio, C.R., 2007. Heme, an iron supply for vibrios pathogenic for fish. Biometals 20, 615-626.

Massé, E., Gottesman, S., 2002. A small RNA regulates the expression of genes involved in iron metabolism in Escherichia coli. Proc. Natl. Acad. Sci. U. S. A. 99, 4620-4625.

Massé, E., Vanderpool, C.K., Gottesman, S., 2005. Effect of RyhB small RNA on global iron use in Escherichia coli. J. Bacteriol. 187, 6962-6971.

Mey, A.R., Craig, S.A., Payne, S.M., 2005a. Characterization of Vibrio cholerae RyhB: the RyhB regulon and role of ryhB in biofilm formation. Infect. Immun. 73, 5706-5719.

Mey, A.R., Wyckoff, E.E., Kanukurthy, V., Fisher, C.R., Payne, S.M., 2005b. Iron and Fur regulation in Vibrio cholerae and the role of Fur in virulence. Infect. Immun. 73, 8167-8178.

Murphy, E.R., Payne, S.M., 2007. RyhB, an iron-responsive small RNA molecule, regulates Shigella dysenteriae virulence. Infect. Immun. $75,3470-3477$

Nguyen, A.T., O’Neill, M.J., Watts, A.M., Robson, C.L., Lamont, I.L., Wilks, A., Oglesby-Sherrouse, A.G., 2014. Adaptation of iron homeostasis pathways by a Pseudomonas aeruginosa pyoverdine mutant in the cystic fibrosis lung. J. Bacteriol. 196, 2265-2276.

Ochsner, U.A., Johnson, Z., Lamont, I.L., Cunliffe, H.E., Vasil, M.L., 1996. Exotoxin A production in Pseudomonas aeruginosa requires the ironregulated pvdS gene encoding an alternative sigma factor. Mol. Microbiol. 21, 1019-1028.

Oglesby-Sherrouse, A.G., Murphy, E.R., 2013. Iron-responsive bacterial small RNAs: variations on a theme. Metallomics 5, 276-286.

Oglesby, A.G., Farrow 3rd, J.M., Lee, J.H., Tomaras, A.P., Greenberg, E.P., Pesci, E.C., Vasil, M.L., 2008. The influence of iron on Pseudomonas aeruginosa physiology: a regulatory link between iron and quorum sensing. J. Biol. Chem. 283, 15558-15567.

Oglesby, A.G., Murphy, E.R., Iyer, V.R., Payne, S.M., 2005. Fur regulates acid resistance in Shigella flexneri via RyhB and ydeP. Mol. Microbiol. 58, $1354-1367$.

Ortega, A.D., Gonzalo-Asensio, J., Garcia-del Portillo, F., 2012. Dynamics of Salmonella small RNA expression in non-growing bacteria located inside eukaryotic cells. RNA Biol. 9, 469-488.

Palyada, K., Threadgill, D., Stintzi, A., 2004. Iron acquisition and regulation in Campylobacter jejuni. J. Bacteriol. 186, 4714-4729.

Payne, S.M., Wyckoff, E.E., Murphy, E.R., Oglesby, A.G., Boulette, M.L., Davies, N.M., 2006. Iron and pathogenesis of Shigella: iron acquisition in the intracellular environment. Biometals 19, 173-180.

Pich, O.Q., Merrell, D.S., 2013. The ferric uptake regulator of Helicobacter pylori: a critical player in the battle for iron and colonization of the stomach. Future Microbiol. 8, 725-738.

Porcheron, G., Garenaux, A., Proulx, J., Sabri, M., Dozois, C.M., 2013. Iron, copper, zinc, and manganese transport and regulation in pathogenic enterobacteria: correlations between strains, site of infection and the relative importance of the different metal transport systems for virulence. Front. Cell. Infect. Microbiol. 3, 90.

Porcheron, G., Habib, R., Houle, S., Caza, M., Lepine, F., Daigle, F., Masse, E., Dozois, C.M., 2014. The small RNA RyhB contributes to siderophore production and virulence of uropathogenic Escherichia coli. Infect. Immun. 82, 5056-5068.

Prevost, K., Salvail, H., Desnoyers, G., Jacques, J.F., Phaneuf, E., Massé, E., 2007. The small RNA RyhB activates the translation of shiA mRNA encoding a permease of shikimate, a compound involved in siderophore synthesis. Mol. Microbiol. 64, 1260-1273.

Reinhart, A., Powell, D.A., Nguyen, A.T., O'Neill, M., Djapgne, L., Wilks, A., Ernst, R.K., Oglesby-Sherrouse, A.G., 2015. The prrF-encoded small regulatory RNAs are required for iron homeostasis and virulence of Pseudomonas aeruginosa. Infect. Immun., http://dx.doi.org/10.1128/ IAI.02707-14.

Salvail, H., Caron, M.P., Belanger, J., Massé, E., 2013. Antagonistic functions between the RNA chaperone Hfq and an sRNA regulate sensitivity to the antibiotic colicin. EMBO J. 32, 2764-2778.

Salvail, H., Massé, E., 2012. Regulating iron storage and metabolism with RNA: an overview of posttranscriptional controls of intracellular iron homeostasis. Wiley Interdiscip. Rev. RNA 3, 26-36.

Santander, J., Golden, G., Wanda, S.Y., Curtiss 3rd, R., 2012. Fur-regulated iron uptake system of Edwardsiella ictaluri and its influence on pathogenesis and immunogenicity in the catfish host. Infect. Immun. 80, 2689-2703.

Sineva, E., Shadrin, A., Rodikova, E.A., Andreeva-Kovalevskaya, Z.I., Protsenko, A.S., Mayorov, S.G., Galaktionova, D.Y., Magelky, E., Solonin, A.S., 2012. Iron regulates expression of Bacillus cereus hemolysin II via global regulator Fur. J. Bacteriol. 194, 3327-3335.

Sun, F., Gao, H., Zhang, Y., Wang, L., Fang, N., Tan, Y., Guo, Z., Xia, P., Zhou, D., Yang, R., 2012. Fur is a repressor of biofilm formation in Yersinia pestis. PLoS ONE 7, e52392. 
Tanabe, T., Funahashi, T., Nakao, H., Maki, J., Yamamoto, S., 2013. The Vibrio parahaemolyticus small RNA RyhB promotes production of the siderophore vibrioferrin by stabilizing the polycistronic mRNA. J. Bacteriol. 195, 3692-3703.

Tobe, T., Yen, H., Takahashi, H., Kagayama, Y., Ogasawara, N., Oshima, T. 2014. Antisense transcription regulates the expression of the enterohemorrhagic Escherichia coli virulence regulatory gene ler in response to the intracellular iron concentration. PLOS ONE 9, e101582.

Torres, V.J., Attia, A.S., Mason, W.J., Hood, M.I., Corbin, B.D., Beasley, F.C., Anderson, K.L., Stauff, D.L., McDonald, W.H., Zimmerman, L.J., Friedman, D.B., Heinrichs, D.E., Dunman, P.M., Skaar, E.P., 2010. Staphylococcus aureus fur regulates the expression of virulence factors that contribute to the pathogenesis of pneumonia. Infect. Immun. 78, $1618-1628$.

Troxell, B., Hassan, H.M., 2013. Transcriptional regulation by Ferric Uptake Regulator (Fur) in pathogenic bacteria. Front. Cell. Infect. Microbiol. 3,59 .

Troxell, B., Sikes, M.L., Fink, R.C., Vazquez-Torres, A., Jones-Carson, J. Hassan, H.M., 2011. Fur negatively regulates hns and is required for the expression of HilA and virulence in Salmonella enterica serovar Typhimurium. J. Bacteriol. 193, 497-505.
Vasileva, D., Janssen, H., Honicke, D., Ehrenreich, A., Bahl, H., 2012. Effect of iron limitation and fur gene inactivation on the transcriptional profile of the strict anaerobe Clostridium acetobutylicum. Microbiology 158, 1918-1929.

Wang, F., Cheng, S., Sun, K., Sun, L., 2008. Molecular analysis of the fur (ferric uptake regulator) gene of a pathogenic Edwardsiella tarda strain. J. Microbiol. 46, 350-355.

Wang, H.R., Hu, Y.H., Zhang, W.W., Sun, L., 2009. Construction of an attenuated Pseudomonas fluorescens strain and evaluation of its potential as a cross-protective vaccine. Vaccine 27, 4047-4055.

Wassenaar, T.M., Gaastra, W., 2001. Bacterial virulence: can we draw the line? FEMS Microbiol. Lett. 201, 1-7.

Wen, Y., Kim, I.H., Son, J.S., Lee, B.H., Kim, K.S., 2012. Iron and quorum sensing coordinately regulate the expression of vulnibactin biosynthesis in Vibrio vulnificus. J. Biol. Chem. 287, 26727-26739.

Wilderman, P.J., Sowa, N.A., FitzGerald, D.J., FitzGerald, P.C., Gottesman, S Ochsner, U.A., Vasil, M.L., 2004. Identification of tandem duplicate regulatory small RNAs in Pseudomonas aeruginosa involved in iron homeostasis. Proc. Natl. Acad. Sci. U. S. A. 101, 9792-9797.

Zhu, C., Ngeleka, M., Potter, A.A., Allan, B.J., 2002. Effect of fur mutation on acid-tolerance response and in vivo virulence of avian septicemic Escherichia coli. Can. J. Microbiol. 48, 458-462. 